\title{
UTILIZAÇÃO DE UM SISTEMA DE AQUISIÇÃO AUTOMÁTICA DE DADOS PARA AVALIAÇÃO DOS NÍVEIS DE RUÍDO DE UM TRATOR AGRÍCOLA DE PNEUS $^{1}$
}

\author{
Paulo Fernando dos Santos Filho ${ }^{2}$, Haroldo Carlos Fernandes ${ }^{3}$, Daniel Marçal de Queiroz ${ }^{3}$, Amaury \\ Paulo de Souza ${ }^{4}$, Arlindo José Camilo ${ }^{5}$
}

\begin{abstract}
RESUMO - Este trabalho foi realizado na Faculdade de Engenharia de Guaratinguetá (FEG-UNESP), SP, no Laboratório de Vibração e Acústica, e numa área experimental localizada no município de Guaratinguetá, com os objetivos de determinar os níveis de ruído emitidos por um trator agrícola executando a operação de gradagem em diferentes velocidades de trabalho e analisar o conforto do operador, comparando os níveis obtidos com os recomendados pelas principais normas vigentes. Na avaliação dos níveis de ruído, utilizaram-se um trator de pneus de 55,2 kW (75 cv) e uma grade destorroadora-niveladora “off-set” de 28 discos. O sistema de aquisição de dados foi constituído por sensores de ruído, condicionadores e amplificadores e um conversor analógicodigital instalados em um microcomputador e embarcados no trator ensaiado. Posteriormente, os dados foram tratados pelo Programa ORIGIN 50. Com base nos resultados, concluiu-se que o sistema trator-implemento emitiu níveis de ruído acima dos limites estipulados pelas normas, em todas as velocidades testadas.
\end{abstract}

Palavras-chave: Ruído, trator e sensores.

\section{UTILIZATION OF A DATA AUTOMATIC ACQUISITION SYSTEM FOR NOISE LEVEL EVALUATION OF AN AGRICULTURAL TRACTOR WITH TIRES}

\begin{abstract}
A study was carried out at the Faculdade de Engenharia de Guaratinguetá -FEG-UNESP, SP, in the acoustics and vibration laboratory as well as in an experimental area located in Guaratinguetá county. The objectives were to determine the noise levels emitted by an agricultural tire tractor during harrowing operation at different speeds and analyze the operator's comfort, by comparing the actual levels with the main effective norms. When evaluating noise levels, a $55.2 \mathrm{~kW}$ (75 hp) tire tractor and an off-set 28-disk harrow were used. The data acquisition system consisted of some noise sensors, conditioners, amplifiers, and a digital-analogical converter plugged to a microcomputer and connected to the tractor tested. Later, the software ORIGIN 50 treated the data. Based on the obtained results, it was concluded that the noise levels emitted by the tractor-implement system exceeded the limits specified by the norms, for all tested speeds.
\end{abstract}

Key words: Noise; tractor and sensors.

\section{INTRODUÇÃO}

O avanço da mecanização agrícola levou, principalmente nas grandes áreas, à substituição da tração animal por máquinas modernas, que têm resolvi- do uma série de problemas no plantio e colheita. No início, os projetos dos tratores estavam mais voltados à redução nos custos de produção e equipamento. Com o desenvolvimento tecnológico, passou-se a buscar o aperfeiçoamento da relação homem-máquina,

\footnotetext{
${ }^{1}$ Recebido para publicação em 19.9.2002 e aceito para publicação em 08.6.2004.

${ }^{2}$ Doutorando em Engenharia Agrícola do Departamento de Engenharia Agrícola da UFV.

${ }^{3}$ Professor Adjunto do Departamento de Engenharia Agrícola da UFV.

${ }^{4}$ Professor Titular do Departamento de Engenharia Florestal da UFV.

${ }^{5}$ Estudante de graduação do Curso de Engenharia Agrícola e Ambiental da UFV.
} 
no sentido de impor ao homem uma carga de trabalho mais suave, visando à diminuição da fadiga. Atualmente, vêm sendo desenvolvidos vários estudos, procurando facilitar a relação do homem com o ambiente de trabalho. Esses estudos envolvem várias outras ciências que, dentro do contexto das disciplinas afins, estão ligadas à organização e à segurança do trabalho, concebendo sistemas de produção mais eficazes dos pontos de vista econômico, técnico e social.

O conforto do tratorista constitui um importante diferencial no aumento da produtividade nas operações agrícolas. As cabines providas de ar-condicionado e poltronas confortáveis favorecem o trabalho dos operadores de máquinas, pois a temperatura elevada, o ruído e vibrações geram desconforto significativo. A diminuição dos níveis de vibração e ruído em veículos agrícolas implicam avanços nos projetos, no sentido de obter um melhor aproveitamento de potência e diminuição de desgaste e de esforços mecânicos.

Schlosser et al. (2000), citados por Schlosser e Debiasi (2002), realizaram uma comparação em termos de nível de ruído à altura do ouvido do operador, considerando o mesmo trator com cabine e sem cabine. Os resultados indicaram que, na ausência de cabines, o ruído ficou acima dos limites considerados salubres para uma jornada de trabalho de oito horas (85 dB). A montagem da cabine reduziu o nível de ruído em, aproximadamente, $5 \mathrm{~dB}$. Além disso, não houve diferenças significativas no nível de ruído com as janelas da cabine abertas, ou fechadas, até a rotação de 2.000 rpm. Nessa rotação, o ruído foi maior com as janelas abertas, o que pode ser explicado em razão de problemas construtivos apresentados pela cabine testada.

Partindo da hipótese de que os tratores agrícolas promovem altos níveis de ruído que podem causar sérios danos à saúde dos operadores, este trabalho teve como objetivos:

- Determinar os níveis de ruído de um trator agrícola em operação de gradagem, em diferentes velocidades de trabalho.

- Analisar o conforto do operador, comparandose os níveis de ruído obtidos com os previstos nas principais normas vigentes.

R. Árvore, Viçosa-MG, v.28, n.3, p. 381-386, 2004

\section{MATERIAL E MÉTODOS}

\subsection{Localização do experimento}

O experimento foi conduzido no município de Guaratinguetá, SP, com altitude média de 537 m, longitude de $22^{\circ} 52^{\prime} 40^{\prime \prime} \mathrm{W}$ (Gr) e latitude de $22^{\circ} 48^{\prime} 43^{\prime \prime}$ S.

\subsection{Trator e implemento}

\subsubsection{Trator}

O trator encontrava-se lastrado, com o tanque, radiador, reservatórios de fluidos de lubrificação e hidráulicos cheios. Os pneus utilizados no ensaio eram de tamanho padronizado para o trator, conforme especificação do fabricante.

Características do motor:

- Marca/modelo: Perkins 4000.

- Ano de fabricação: 1999.

- Tipo: Diesel, injeção direta, 4 tempos.

- Cilindros: 4, verticais em linha.

- Cilindrada: $4.000 \mathrm{~cm}^{3}$.

- Relação de compressão: 16:1.

- Potência no motor a 2.200 rpm: 55,2 kW (75 cv).

Transmissão de potência:

- Embreagem: independentemente, 2 discos secos, 254/305 mm de diâmetro e acionamento mecânico por pedal.

- Caixa de câmbio: sincronizada, com 12 marchas para frente e 4 para trás.

- Diferencial: com bloqueio mecânico acionado por pedal.

- Barra de tração: oscilante.

Tomada de potência:

- 35 mm, com 6 estrias.

- Potência máxima (2.200rpm): 49,25 kW (67cv).

- Potência a 540 rpm (1.680 rpm no motor): 45,5 $\mathrm{kW}(62,0 \mathrm{cv})$.

Outros dados:

- Tração dianteira auxiliar. 
- Rodagem diagonal: Rodagem dianteira 12,4-24 R1, com pressão de insuflação de 96,5 kPa (14 psi); traseira 18,4-30 R1, com pressão de 110,3 kPa (16 psi), em boas condições.

- Massa sem lastro: $2.553 \mathrm{~kg}$.

- Massa com lastro: $3.673 \mathrm{~kg}$.

- Sem cabine e estrutura de proteção contra capotamento, com toldo.

\subsubsection{Implemento}

O implemento utilizado foi uma grade destorroadoraniveladora "off-set", com 28 discos de 0,5 x 0,0035 m (20" x 3,5 mm) e massa aproximada de $666 \mathrm{~kg}$. Alargura de trabalho foi de $2,6 \mathrm{~m}$.

\subsection{Caracterização da área}

O solo foi classificado como Latossolo Vermelho-Amarelo de textura franco-argilosa, de acordo com o levantamento de reconhecimento dos solos do Estado de São Paulo (Brasil, 1960). Antes da gradagem, foi realizada uma aração com um arado de discos de $3 \mathrm{x}$ 26 ", à profundidade de $0,18 \mathrm{~m}$. A gradagem foi realizada no sentido longitudinal da aração. O experimento foi conduzido com o solo estando com um teor de água de $26 \%$, determinado pelo método gravimétrico padrão com base na massa de solo secado em estufa à temperatura de $105-110^{\circ} \mathrm{C}$ até a obtenção de uma massa constante, conforme Embrapa (1997).

\subsection{Velocidade de deslocamento}

As velocidades utilizadas nos testes foram 1,39 $\mathrm{m} / \mathrm{s} ; 1,67 \mathrm{~m} / \mathrm{s} ;$ e $1,95 \mathrm{~m} / \mathrm{s}$ na $1^{\mathrm{a}}$, 2ª e $3^{\text {a }}$ marchas, respectivamente. A velocidade real foi determinada, medindose, com um cronômetro digital, o tempo gasto para percorrer $30 \mathrm{~m}$. A rotação do motor do trator foi de 1.800 rpm em todos os testes, com o trator em movimento sobre o solo arado.

\subsection{Dados climatológicos}

Os dados de umidade relativa e temperatura do ar e velocidade do vento foram obtidos dos registros da Estação Meteorológica de Guaratinguetá. No dia do experimento, a umidade relativa era de $40 \%$, a temperatura ambiente de $28,2{ }^{\circ} \mathrm{C}$ e a velocidade do vento de $3,2 \mathrm{~m} / \mathrm{s}$.
Segundo a norma NBR 9999 (ABNT, 1987), a temperatura ambiente, na posição de medição, deve estar entre -5 e $30^{\circ} \mathrm{C}$, e a velocidade do vento não deve ultrapassar $5 \mathrm{~m} / \mathrm{s}$ em relação ao solo.

\subsection{Montagem do experimento}

As medições de ruído foram realizadas ao mesmo tempo em três diferentes velocidades do sistema trator-implemento, sendo em cada velocidade realizada uma passada com o tempo total de $60 \mathrm{~s}$. A freqüência de aquisição foi de 400 pontos por segundo, gerando 24.000 pontos, para cada velocidade. Na análise estatística dos sinais, utilizou-se a estatística descritiva para calcular a média, o desvio-padrão e a regressão linear dos dados coletados em cada velocidade de deslocamento do sistema trator-implemento.

O sistema trator-implemento foi testado em condições de campo, numa área relativamente plana, sem obstáculos e declives, os quais poderiam ocasionar deflexão do som ou amplitudes vibratórias anômalas.

A escala utilizada para medida do ruído do trator foi o decibel (dBA). As determinações dos níveis de ruído foram baseadas na NBR 9999 (ABNT, 1987) e na metodologia usada por Vitória (2000).

\subsection{Equipamentos para medição dos níveis de ruído}

Para medir o NPS (nível de pressão sonora) foi utilizado o decibelímetro da marca MINIPA, composto basicamente de um microfone, amplificadores, circuitos integradores, filtros, poderadores e indicadores de leitura. A resposta de freqüência considerada foi uma aproximação da resposta do ouvido humano, à qual se aplicaram a ponderação A e a resposta lenta. Os sinais analógicos foram digitalizados e armazenados pelo sistema de aquisição constituído de um sensor, condicionador e amplificadores e, posteriormente, enviados para um conversor analógico-digital (modelo LYNX CAD 10/26; 12 bits; $+10 \mathrm{~V}$ e $-10 \mathrm{~V}$ e taxa de conversão A/D $40 \mathrm{kHz}$ ) usado como interface com um computador PC-XT.

Os equipamentos foram alimentados por uma bateria de $12 \mathrm{~V}$ ligada a um inversor de tensão (12 V-DC/110 V- AC). Para transformar volts em dBA, utilizou-se a relação do sensor $10 \mathrm{mV} / \mathrm{dBA}$, sendo, logo em seguida, obtidas as médias de 24.000 pontos para cada velocidade. O decibelímetro foi instalado no mesmo lado do tubo de descarga, onde se supunha ser o lado

R. Árvore, Viçosa-MG, v.28, n.3, p. 381-386, 2004 
com maiores níveis de ruído (LIMA, 1998). Para calibrar o sensor, fez-se um ajuste interno, regulando a chave de funções para a posição "cal 94 dB", a chave "response" para a posição "F" e a chave "range" para a posição "HI". Na calibração, empregou-se um sinal senoidal de $1.000 \mathrm{~Hz}$.

\section{RESULTADOS E DISCUSSÃO}

Antes de efetuar as medições dos níveis de pressão sonora, considerou-se a influência da grandeza mencionada como ruído de fundo, isto é, o ruído ambiental gerado por outras fontes que não o objeto de estudo.

A NBR 9999 (ABNT, 1987) estabelece que o nível de ruído de fundo (ruído ambiente) deva estar a pelo menos $10 \mathrm{dBA}$ abaixo do registrado durante o ensaio. Em todos os resultados, não foi necessário corrigir a medida de ruído.

\subsection{Níveis de ruído durante a gradagem}

Nas Figuras 1, 2 e 3, apresentam-se os níveis de ruído obtidos com o trator trabalhando a três velocidades. Observa-se, nessas figuras, que o maior nível de ruído ocorreu na terceira marcha. Resultado semelhante foi encontrado por Vitória (2000), que avaliou os níveis de ruído emitido por tratores executando diferentes operações agrícolas. Esse autor constatou que, numa mesma rotação do motor, o trator apresentou as maiores médias dos níveis de ruído, quando se utilizaram as maiores velocidades e maiores profundidades.

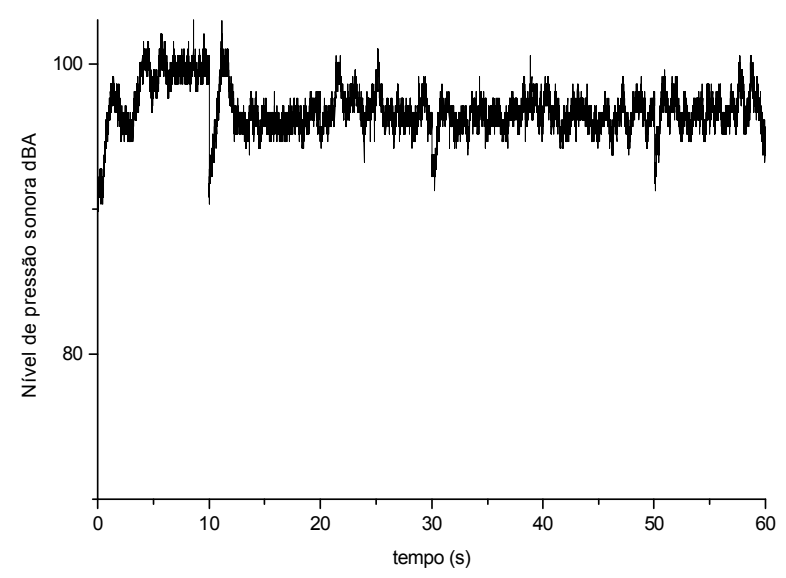

Figura 1-Registro dos níveis de ruído (terceira marcha). Figure 1 - Noise level registration (third gear).

R. Árvore, Viçosa-MG, v.28, n.3, p. 381-386, 2004

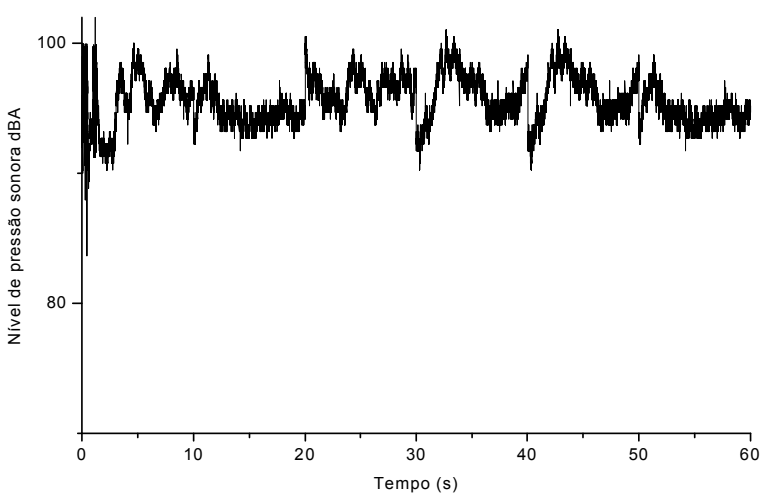

Figura 2 - Registro dos níveis de ruído (segunda marcha). Figure 2 - Noise level registration (segund gear).

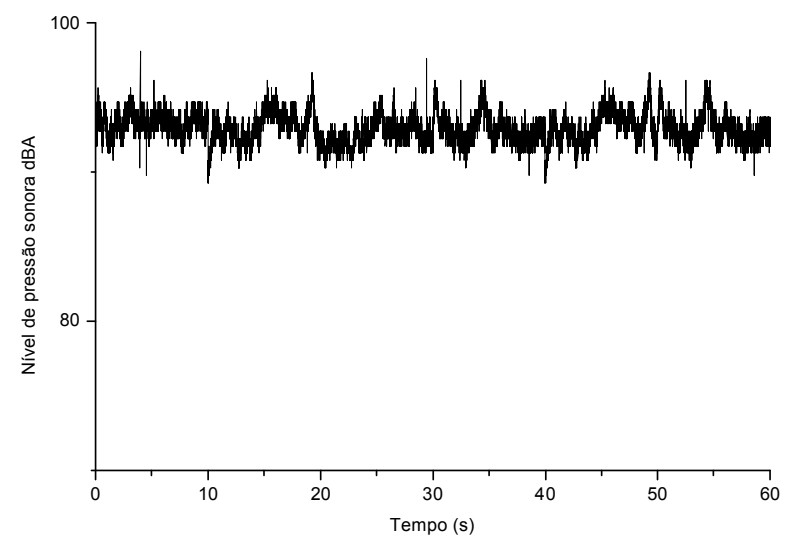

Figura 3 - Registro dos níveis de ruído (primeira marcha). Figure 3 - Noise level registration (first gear).

Fernandes (1991) observou aumento nos níveis de ruído com o aumento na velocidade de deslocamento do trator, principalmente quando se utilizavam implementos tracionados pela barra de tração. Nesse mesmo trabalho foram encontrados níveis de ruído apresentando altos valores, com uma média de 96,07 $\mathrm{dBA}$, o que permitiria apenas $1 \mathrm{~h} 15$ de trabalho diário do tratorista (NR-15, CLT).

$\mathrm{Na}$ Figura 4, mostra-se a linha de tendência linear, obtida da correlação das médias do ruído com as respectivas velocidades. Apresentando um $\mathrm{R}^{2}$ de 0,93 , caracteriza-se uma boa relação funcional entre o nível médio de ruído e a velocidade do trator. Esse aumento no nível de ruído em função de maiores velocidades pode ter sido devido à maior vibração dos componentes do trator e, também, do rolamento dos discos da grade. 


\subsection{Análise estatística dos níveis de ruído nas três marchas em estudo}

Nas Figuras 5, 6 e 7, caracterizam-se a distribuição de freqüência de ocorrência dos eventos, nas três marchas. A terceira marcha apresentou uma média de 96,761dBA, com um desvio-padrão de $\pm 1,584$ dBA, enquanto a segunda marcha, uma média de 95,407 dBA, com um desvio-padrão de $\pm 1,641 \mathrm{dBA}$, e a primeira marcha, 92,870 dBA, com desvio-padrão de \pm 0,961 dBA. A análise dos sinais evidenciou valores máximos e mínimos de 104 e 89,9 dBA, na terceira marcha; 101,05; e 90,25 dBA, na segunda marcha; e 98,15; e $89,30 \mathrm{dBA}$, na primeira marcha. Todos os valores encontraram-se acima do nível de $85 \mathrm{dBA}$, permitido pala NR-15 (CLT). A análise foi realizada pelo Programa ORIGIN 50.

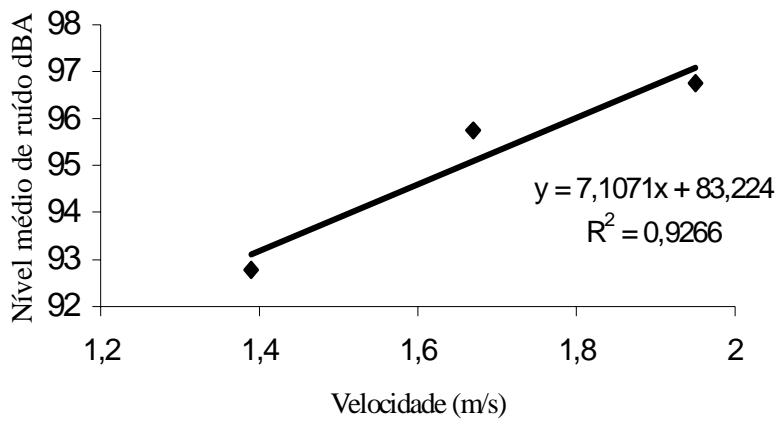

Figura 4 - Linha de tendência entre o nível de ruído e a velocidade.

Figure 4 - Tendency line between the noise level and the speed.

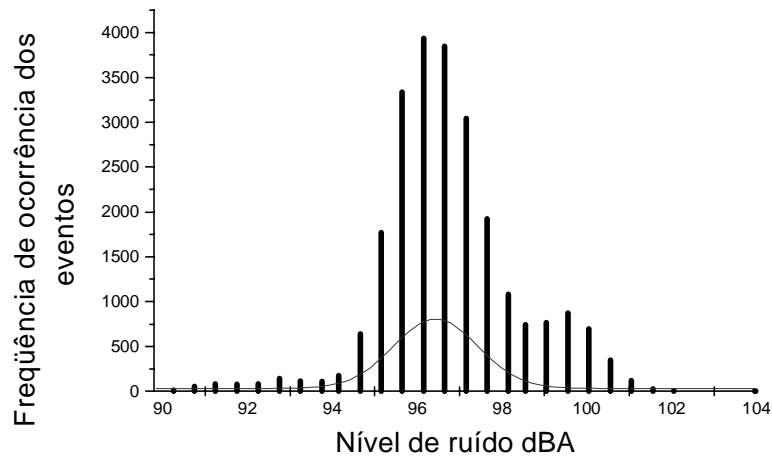

Figura 5 - Análise da distribuição de freqüência dos níveis de ruído (terceira marcha).

Figure 5 - Analysis of the distribution of frequency of the noise levels (third gear).

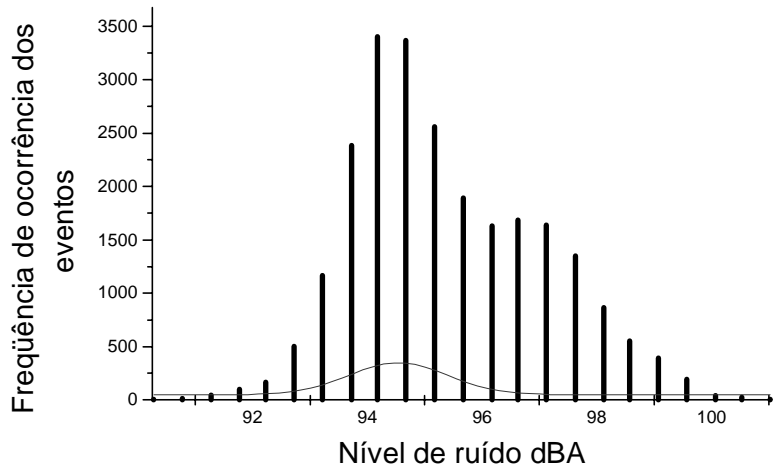

Figura 6 - Análise da distribuição de freqüência dos níveis de ruído (segunda marcha).

Figure 6-Analysis of the distribution of frequency of the noise levels (second gear).

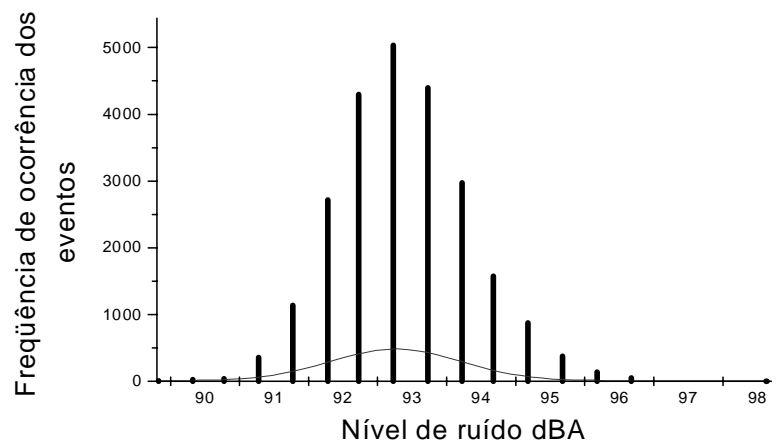

Figura 7 - Análise da distribuição de freqüência dos níveis de ruído (primeira marcha).

Figure 7 - Analysis of the distribution of frequency of the noise levels (first gear).

\subsection{Comparação dos níveis de ruído obtidos durante a gradagem com os valores estabelecidos pelas principais normas técnicas}

Os valores médios dos níveis de ruído foram bastante elevados, causando um grande desconforto ao operador. Esses valores ultrapassam os limites estipulados pela Portaria no 3.214/78-NR15-CLT, que determina um limite de 85 dBA para uma exposição máxima diária de oito horas, sem protetores auriculares, com a ressalva de que esses resultados se equiparam aos encontrados por Fernandes et al. (1991). Esses altos valores médios provavelmente foram devidos ao ruído causado pela movimentação da grade, so-

R. Árvore, Viçosa-MG, v.28, n.3, p. 381-386, 2004 
mado ao já existente do trator agrícola. No Quadro 1, apresenta-se o tempo máximo de exposição que seria permitido por essa portaria.

Quadro 1 - Tempo de exposição diária máxima permissível nas médias de ruído encontradas no conjunto tratorimplemento, de acordo com a Portaria no 3.214/ 78 NR 15-CLT

Table 1 - Time of maximum daily exhibition allowvable for the noise averages found in the group tractorimplement, in agreement with the law nㅇ 3.214/ 78 NR 15-CLT

\begin{tabular}{ccr}
\hline $\begin{array}{c}\text { Velocidade de } \\
\text { deslocamento } \\
\text { do conjunto } \\
\text { trator-implemento } \\
\text { (m/s) }\end{array}$ & $\begin{array}{c}\text { Níveis de ruído } \\
\text { médio encontrados } \\
\text { dBA }\end{array}$ & $\begin{array}{r}\text { Tempo máximo } \\
\text { de exposição } \\
\text { (minuto) }\end{array}$ \\
\hline $\begin{array}{c}\text { 1,95 (terceira marcha) } \\
\text { 1,67 (segunda marcha) }\end{array}$ & 96,761 & 75 \\
1,39 (primeira marcha) & 92,870 & 105 \\
\hline
\end{tabular}

\section{CONCLUSÕES}

De acordo com os resultados experimentais, verificou-se que:

- O sistema trator-implemento emitiu níveis de ruído acima dos limites estipulados pela norma NB 95, como também acima do limite de 85 dBA para oito horas de exposição diária, estabelecido pela NR-15 (CLT), sendo a terceira marcha a que emitiu o maior nível de ruído.

- Apesar de não ter sido feito um teste audiométrico, os níveis de ruído medidos em todas as velocidades de deslocamento do trator indicaram uma condição de trabalho desconfortável para o tratorista, proporcionando-lhe risco de hipoacusia.

\section{REFERÊNCIAS BIBLIOGRÁFICAS}

\section{ASSOCIAÇÃO BRASILEIRA DE NORMAS} TÉCNICAS - ABNT. NBR 9999; medição do nível de ruído, no posto de operação de tratores e máquinas agrícolas. Rio de Janeiro: 1987. 21 p.
Empresa Brasileira de Pesquisa Agropercuária. Serviço Nacional de Levantamento e Conservação de Solos. Manual de métodos de análise de solo. Rio de Janeiro: 1997. 212 p.

FERNANDES, J.C.; LANÇAS, K.P.; SANTOS, J.E.G. O ruído em tratores industriais. Parte II: perda de audição em operadores. In: CONGRESSO BRASILEIRO DE ENGENHARIAAGRÍCOLA, 20., 1991, Londrina. Anais ... Londrina: SBEA, 1991. p. 343-350.

FERNANDES, J.C. Avaliação dos níveis de ruído em tratores agrícolas e seus efeitos sobre o operador. 1991. 192f. Tese (Doutorado em Energia na Agricultura) Universidade Estadual Paulista, Botucatu, 1991.

\section{LIMA, J.S.S. Avaliação da força de arraste, compactação do solo e fatores ergonômicos num sistema de colheita de madeira utilizando os tratores “Feller-Buncher" e “Skidder". 1998. 128 \\ f. Tese (Doutorado em Ciência Florestal) - Universidade Federal de Viçosa, Viçosa, 1998.}

BRASIL. Ministério da Agricultura. Centro Nacional de Ensino e Pesquisas Agronômicas.

Levantamento de reconhecimento dos solos do estado de São Paulo. Rio de Janeiro: Comissão de Solos, 1960. 628 p (Boletim do Serviço Nacional de Pesquisas Agronômicas, 12).

SCHLOSSER, J. F.; DEBIASI, H. Conforto, preocupação com o operador. Revista Cultivar Máquinas. n. 1., p. 3-9, 2002. (Caderno Técnico).

VITÓRIA, E.L. Avaliação dos níveis de ruído emitido por tratores em diferentes operações agrícolas. 2000. $76 \mathrm{f}$. Dissertação (Mestrado em Engenharia Agrícola) Universidade Federal de Viçosa, Viçosa, 2000. 